\title{
A case report of ocular ischemic syndrome with neovascular glaucoma secondary to Moya-Moya disease
}

\author{
Pranathi $\mathrm{B}^{1}$, Jyotsna Myneni ${ }^{1}$, Sharada Bhagawat ${ }^{1}$, and Manaswini Priya Varanasi ${ }^{1, *}$ \\ ${ }^{1}$ Department of Ophthalmology, Krishna Institute of Medical Sciences, Minister Road, Secunderabad-500003, Telangana, India
}

\begin{abstract}
We present a rare case of ocular ischemic syndrome with neovascular glaucoma secondary to Moya-Moya disease in a 49-year-old male patient with complaints of headache, giddiness and gradual diminution of vision in his left eye. A detailed ophthalmic examination followed by investigations of Optical coherence tomography and fundus fluorescein angiography along with carotid doppler and magnetic resonance angiography with Diamox intervention was performed and a diagnosis of ocular ischemic syndrome secondary to Moya-Moya disease has been made. The patient was advised pan retinal photocoagulation in both eyes with intravitreal ranibizumab injection in the left eye for macular edema under guarded visual prognosis. In spite of treatment with three sittings of pan retinal photocoagulation along with intravitreal ranibizumab, the patient's vision deteriorated. It is one of the cases of ocular ischemic syndrome where there is no visual improvement in spite of treatment as it is secondary to a cause like Moya-Moya disease.
\end{abstract}

Keywords: ischemic syndrome; ocular; Moya-Moya disease; neovascularization

*Corresponding author: Dr. Varanasi Manaswini Priya, Department of Ophthalmology, Krishna Institute of Medical Sciences, Minister Road, Secunderabad-500003, Telangana, India. Tel.:+91 9676954619; Email: manaswinipriya1994@gmail.com

Received 13 August 2021; Revised 16 September 2021; Accepted 21 September 2021; Published 27 September 2021

Citation: Pranathi B, Myneni J, Bhagawat S, Varanasi MP. A case report of ocular ischemic syndrome with neovascular glaucoma secondary to Moya-Moya disease. J Med Sci Res. 2021; 9(4):237241. DOI: http://dx.doi.org/10.17727/JMSR.2021/9-37

Copyright: (C) 2021 Pranathi B et al. Published by KIMS Foundation and Research Center. This is an open-access article distributed under the terms of the Creative Commons Attribution License, which permits unrestricted use, distribution, and reproduction in any medium, provided the original author and source are credited.

\section{Introduction}

Ocular ischemic syndrome is a rare condition, which is caused by ocular hypoperfusion due to stenosis or occlusion of the common or internal carotid arteries. Ocular ischemic syndrome is manifested as visual loss, orbital pain and, frequently, changes of the visual field, and various anterior and posterior segment signs [1]. Moya-Moya disease is one of the rare causes of ocular ischemic syndrome. Moya-Moya disease (MMD) is a chronic occlusive cerebrovascular disorder affecting the terminal portions of the bilateral internal carotid arteries or proximal portions of the anterior and middle cerebral arteries. The progressive ischemia results in the formation of a collateral vascular network in the base of the brain [2]. 
A 49-year-old male presented with complaints of diminution of vision, giddiness and headache. After meticulous history taking followed by detailed examination and thorough investigations, we have diagnosed the patient as having ocular ischemic syndrome in both eyes secondary to MMD with neovascular glaucoma in his left eye. In spite of treatment with thee sittings of pan retinal photocoagulation with intravitreal ranibizumab, the patient's vision couldn't be salvaged. We present the following case in accordance with the Care reporting checklist.

\section{Case presentation}

A 49-year-old male patient presented to the OPD with complaints of headache, giddiness and gradual diminution of vision in his left eye for one week. It was painless and gradually progressive loss of vision involving the peripheral visual fields. He had a history of giddiness on getting up from lying down, tingling and numbness of both upper and lower limbs associated with intermittent left sided headaches. He was non diabetic, non-hypertensive. On initial examination, his pulse rate was $88 \mathrm{bpm}$, blood pressure was $160 / 100 \mathrm{mmHg}$, body temperature of $99 \mathrm{~F}$. His random blood sugar was $135 \mathrm{mg} / \mathrm{dl}$. On ophthalmic examination, his best corrected visual acuity in his right eye was $6 / 9$ and it was $6 / 18$ in his left eye. His near vision was N8 in both eyes and his color vision was $1 / 17$ in both eyes. On applanation tonometry, his intraocular pressure was $15 \mathrm{mmHg}$ in his right eye and $24 \mathrm{mmHg}$ in his left eye. On slit lamp examination, both lids, conjunctiva and cornea were normal. There was no evidence of any cells and flare in the anterior chamber in both eyes. Patient had neovascularization of iris in the left eye. Lens showed nuclear cataract of grade 2 with posterior subcapsular cataract in both eyes. The pupil of the left eye showed RAPD grade 4 in his left eye. Undilated fundus examination revealed neovascularization at disc with vitreous florets and old vitreous hemorrhage in his right eye and neovascularization of disc with dense hemorrhage over the disc with vitreous strands in the left eye. A visual perimetry was ordered and it showed circumferential field defects in both eyes (Figure 1).

The optical coherence tomography was normal in his right eye and the left eye showed macular edema. He was called for a review after one week for fundus fluorescein angiography (FFA) after starting him on timolol $0.5 \%$ eye drops twice daily in his left eye. In the next visit, his intraocular pressure (IOP) was 10 $\mathrm{mmHg}$ in the right eye and $16 \mathrm{mmHg}$ in the left eye.
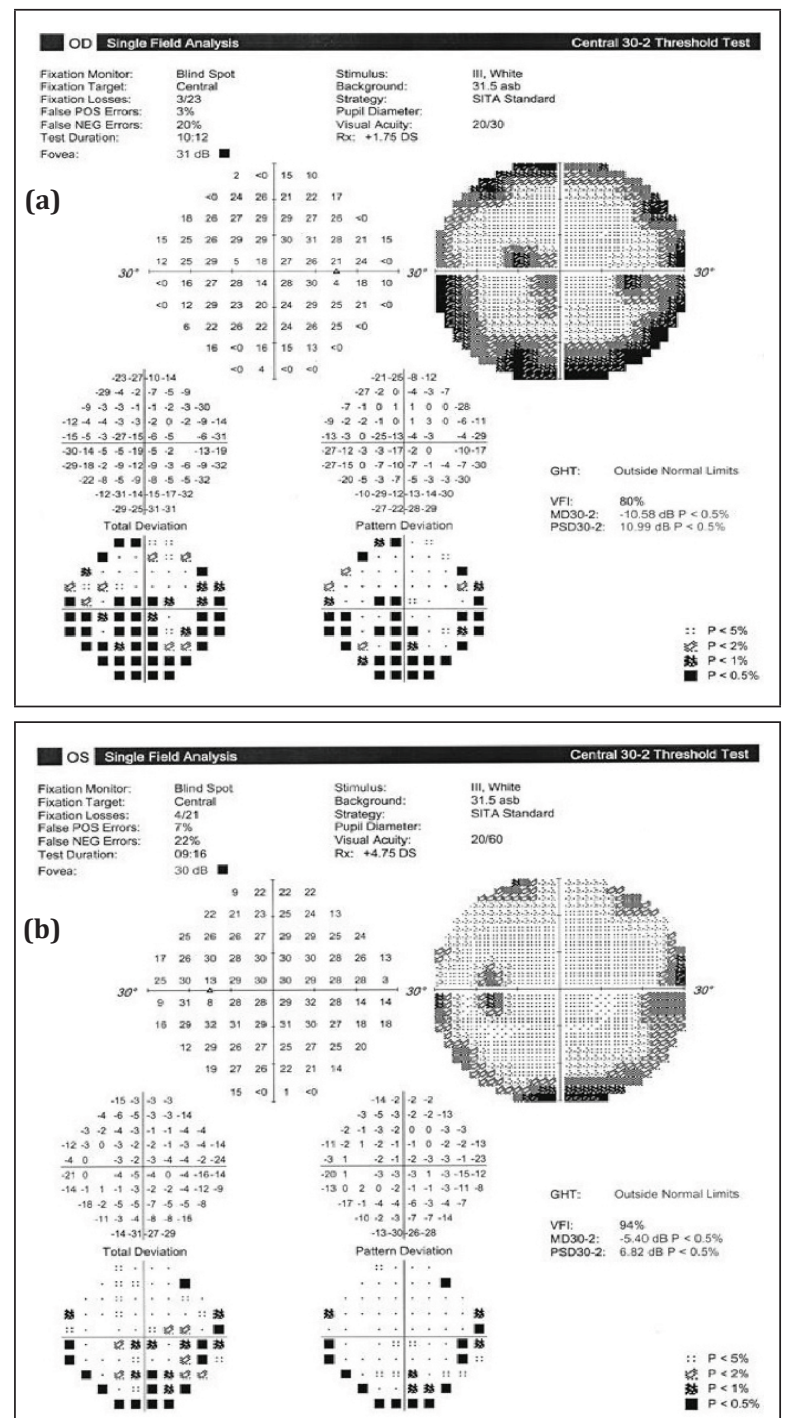

Figure 1a, b: Visual perimetry showing circumferential field defects.

On dilated fundus examination, the right eye showed neovascularization at disc with vitreous florets and the left eye showed neovascularization at disc with signs of dispersed vitritis and pre-discal hemorrhage (Figure 2).

FFA (Figure 3) was performed which showed increased arm-retina circulation time with $58 \mathrm{sec} 18$ milliseconds.

He was advised on the carotid doppler and magnetic resonance angiography (MRA) brain with Diamox 
Spect scan which showed hypoperfusion at the left temporal and temporoparietal regions with further deterioration following Diamox intervention which is suggestive of poor vascular reserve. Magnetic resonance imaging (MRI) with MRA showed bilateral internal carotid artery stenosis with right cerebral hemispheric infarct with remote infarcts on the left side. His blood pressure was $150 / 90 \mathrm{mmHg}$ and the random blood sugar was $121 \mathrm{mg} / \mathrm{dl}$. He was diagnosed as a de novo hypertensive and was started on antihypertensive medications.

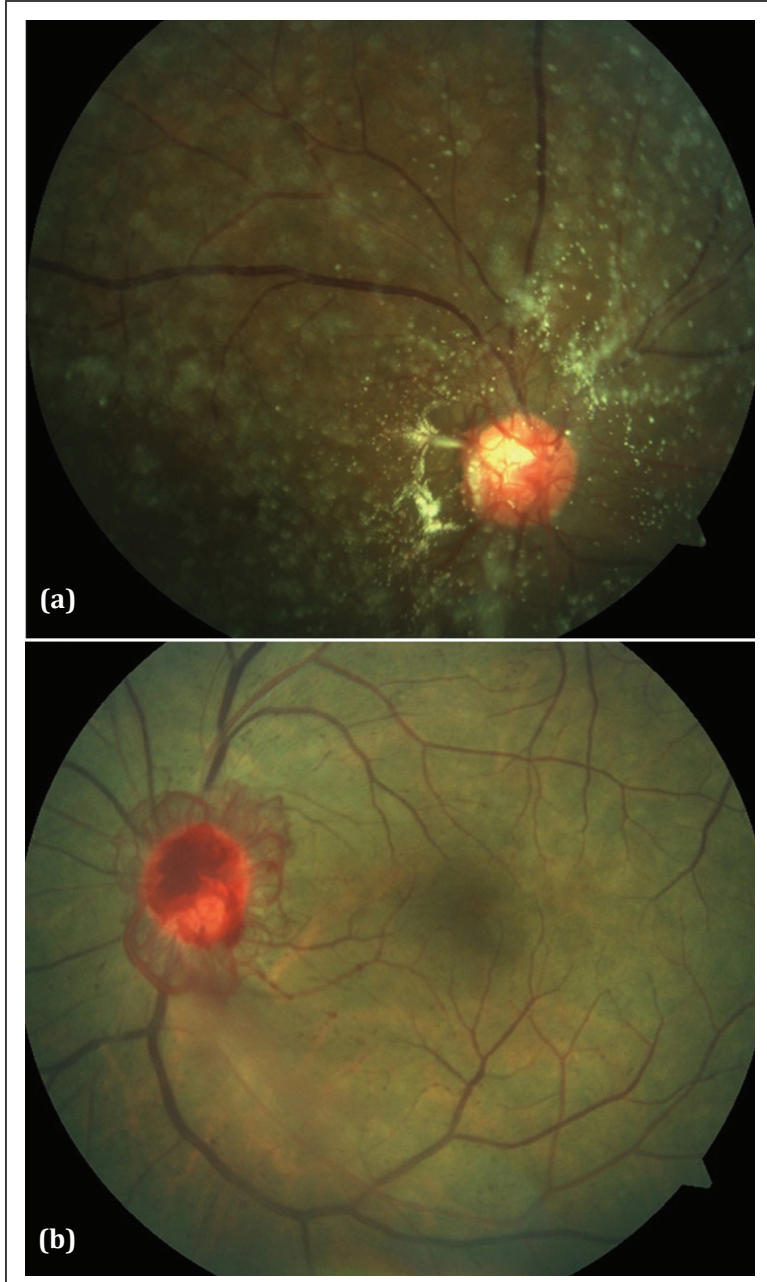

Figure 2a,b: Fundus photo with neovascularization of disc BE with dispersed vitritis.

A diagnosis of MMD with bilateral ocular ischemic syndrome with neovascular glaucoma in the left eye was made. The patient was advised pan retinal photocoagulation for ischemic changes in both eyes along with intravitreal ranibizumab injection in the left eye for NVE with macular edema under guarded visual prognosis. An integrated opinion of neurosurgery, cardiology departments was taken and the patient was started on aspirin $75 \mathrm{mg}$ once a day with rosuvastatin $20 \mathrm{mg}$ once a day. In spite of treatment with 3 sittings of PRP and intravitreal ranibizumab injection, the patient's vision deteriorated in the left eye which came down to finger counting at one meter without any improvement with the pinhole. His target IOP was maintained at $10 \mathrm{mmHg}$ in the right eye and $18 \mathrm{mmHg}$ in the left eye. Patient has been explained about his guarded visual prognosis and asked to continue his followup visits to monitor the other eye involvement. This study conformed to the provisions of the declaration of Helsinki (as revised in 2013).

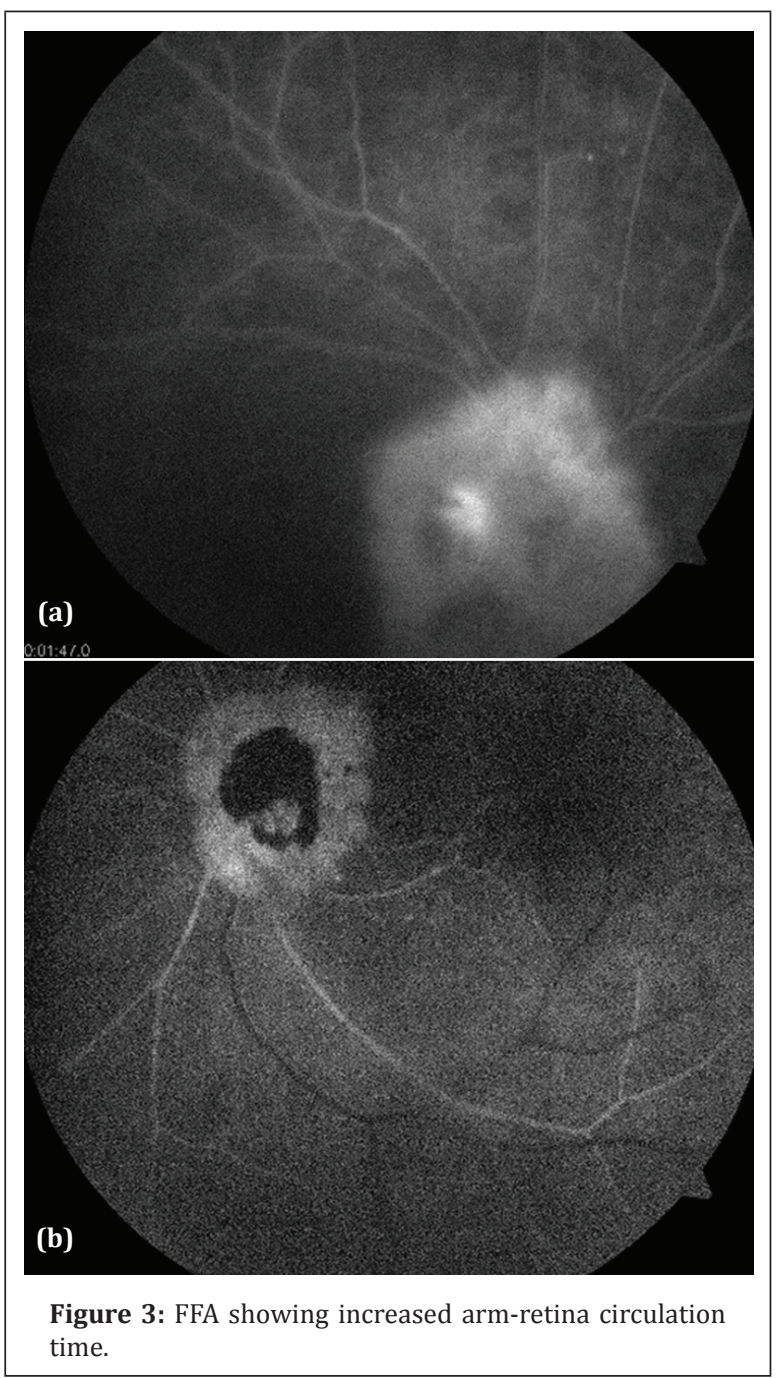

\section{Discussion}

At the time of the patient's presentation, on fundus examination, our initial suspicion was that of a severe proliferative diabetic retinopathy or an ischemic 
central retinal vein occlusion. Investigations of random blood sugar and HBA1C were within normal limits ruling out diabetic retinopathy. His history of neurological complaints has given us a suspicion of ischemic pathology within the brain. A detailed neurological evaluation has confirmed the cerebral ischemia and strengthened our diagnosis of ocular ischemic syndrome.

Ocular ischemic syndrome (OIS) is a condition associated with carotid artery occlusion that leads to ocular hypoperfusion. OIS is a severe form of chronic ischemia of the anterior, posterior segments of the eye, and other orbital structures supplied by the ophthalmic artery [1]. Signs of anterior segment ischemia (conjunctival chemosis and congestion, a mild inflammatory reaction in the anterior chamber, presence of neovascularization, fixed semi-dilated pupil) coupled with posterior segment ischemic signs (retinal opacification with no cherry red spot, retinal hemorrhages extending up to the mid-periphery) immediately point to the diagnosis of ocular ischemic syndrome [2]. Visual loss in the affected eye is present in over $90 \%$ of patients with OIS. It is usually related to chronic or acute retinal ischemia or damage to the optic nerve due to secondary glaucoma. In $67 \%$ of the patients, visual loss occurs gradually over a few weeks or months, in $12 \%$ it occurs over a period of days, and in another $12 \%$ the loss is sudden over a period of minutes or seconds [3]. Visual loss is a consequence of chronic retinal and choroidal ischemia is the excessive production of vascular endothelial growth factor (VEGF). VEGF acts on epithelial cells to result in new blood vessel growth. This neovascularization occurs in the iris and at the iridocorneal angle in the anterior segment.

MMD is a chronic occlusive cerebrovascular disorder affecting the terminal portions of the bilateral internal carotid arteries or proximal portions of the anterior and middle cerebral arteries. The progressive ischemia results in the formation of a collateral vascular network in the base of the brain. The term "Moya-Moya" is a Japanese word which means a hazy puff of smoke, used to describe the abnormal appearance of the vascular network seen in angiograms of patients with MMD $[4,5]$. It is one of the rarer causes of ocular ischemic syndrome. Ocular manifestations in MMD are uncommon, but several case reports have described varying ocular manifestations including amaurosis fugax, central retinal artery occlusion, morning glory disc anomaly, and ocular ischemic syndrome, retinochoroidal coloboma, microphthalmos, iris hypoplasia, Peter's anomaly and chorioretinal atrophy $[6,7]$.

Differential diagnosis of ocular ischemic syndrome includes diabetic retinopathy and moderate central retinal vein occlusion. Pan retinal photocoagulation is indicated in patients with iris and posterior segment neovascularization to prevent development of secondary neovascular glaucoma and intraocular hemorrhages. Full peripheral retinal ablation 3000-5000 burns of 200-500 $\mu \mathrm{m}$ spot size should be used [8]. However, it is effective in only 36\% of treated eyes with OIS because choroidal ischemia alone with no retinal ischemia may be sufficient to induce neovascularization. Neovascular glaucoma can occur secondary to ocular ischemic syndrome caused by Moya-Moya disease [9]. The visual prognosis was worse for eyes with rubeosis iridis on initial presentation. Eighty-two percent of eyes with rubeosis at or within three months of presentation had no better than count fingers vision at one-year follow up [10].

\section{Conclusion}

We would conclude that ocular ischemic syndrome is a rare condition which can be easily misdiagnosed in cases without a high index of suspicion. Even in the cases that are thoroughly investigated and followed up, the rate of visual recovery is quite less due to the chronic ischemic damage to the retinal layers.

\section{Conflict of interest}

Authors declare no conflict of interest.

\section{References}

[1] Balamurugan S, Manohar BB, Bharat G, Annamalai O, Mikeen $\mathrm{S}$, et al. Ocular ischemic syndrome. TNOA J Ophthal Sci Res. 2020; 58:20.

[2] Jayesh AV, Zoramthara Z, Mangat RD, Amod G. Ocular ischemic syndrome, Indian J Ophthal. 2014; 62(5):658660.

[3] Terelak-Borys B, Skonieczna K, Grabska-Liberek I. Ocular ischemic syndrome - A systematic review. Med Sci Monit. 2012; 18(8):138-144.

[4] Bakri SJ, Siker D, Masaryk T, Luciano MG, Traboulsi EI. Ocular malformations, moyamoya disease, and midline cranial defects: a distinct syndrome, American J Ophthal. 1999; 127(3):356-357.

[5] Witmer MT, Levy R, Yohay K, Kiss S. ophthalmic artery ischemic syndrome associated with neurofibromatosis and Moyamoya syndrome. JAMA Ophthalmol. 2013; 131(4):538539. 
[6] Evangelia P, Lucia S, George NP. Ocular ischemic syndrome presenting as retinal vasculitis in a patient with moyamoya syndrome. Retinal Cases and Brief Reports. 2015; 9(2):170172.

[7] John D, Muthusamy K, Bandla B, Sudhakar SV, Thomas M. Ocular features and visual outcome in children with Moyamoya disease and Moyamoya syndrome: A case series. J Clin Diagn Res. 2016; 10(5):NR01-NR4.

[8] Malhotra R, Gregory-Evans K. Management of ocular ischaemic syndrome. British J Ophthal. 2000; 84:14281431.

[9] A case of neovascular glaucoma secondary to ocular ischemic syndrome in a patient with Moyamoya disease. Seung Min Lee, Ji Woong Lee, Department of Ophthalmology, Pusan National University School of Medicine, Busan, Korea.

[10] Barrall JL, Summers CG. Ocular ischemic syndrome in a child with Moyamoya disease and neurofibromatosis. Surv Ophthalmol. 1996; 40(6):500-504. 